\title{
New approach to the organization of entrances to cities using ITS
}

\author{
Sergey Dorokhin ${ }^{1, *}$, Evgenii Chirkov $^{1}$, and Sergey Postavnichii ${ }^{1}$ \\ ${ }^{1}$ Voronezh State University of Forestry and Technologies named after G. F. Morozov, Voronezh, \\ 394087, Russia
}

\begin{abstract}
The article analyses the use of intelligent transport systems, as a result of the study, the authors found that in large cities today, intelligent entries are not used much, which reduces the load on the entry areas. The authors propose an algorithm for organizing entry on the example of Voronezh and propose a method for passing vehicles on the main and secondary roads, as a result of which the efficiency of organizing traffic to large cities is obtained.
\end{abstract}

\section{Introduction}

Today, the largest and largest cities in Russia are faced with the problem of oversaturation of the road network with road transport. A high level of motorization leads to a decrease in the efficiency of the functioning of roads and streets, which leads to a deterioration of environmental and economic indicators, the quality of transport services and travel comfort are reduced. Although the level of motorization in Russia is much lower compared to developed European countries, our cities are already experiencing difficulties in satisfying the demand of residents on the road network, which is not observed in countries with motorization above 500 cars per 1000 people.

It is possible to level the steadily growing number of cars in two ways: architectural and planning events and traffic management. The first is fraught with grandiose timeconsuming and expensive projects, which undoubtedly give the desired effect, but valuable for a large investment of time and resources. And although Russia does not suffer from a lack of territory for construction, the experience of foreign countries (such as Japan, where land plots are very scarce) has shown that it is possible to increase the efficiency of the road network by organizing traffic using intelligent transport systems. The organization of traffic as an event to increase the effective indicators of the road network is less costly and has the best ratio according to the criterion of benefits / costs. By applying the achievements of ITS, it presents great opportunities for the implementation of the most highly efficient traffic management systems, especially when managing entrances to large cities.

\footnotetext{
*Corresponding author: dsvvrn@yandex.ru
} 


\section{Analysis of city entry management systems}

Exit control methods are designed to maintain a balance between highway throughput and demand by maintaining optimal highway operation. Congresses are used to regulate the passage on a specific ramp, for example, the introduction of a partial restriction on travel (on a schedule) or, possibly, a permanent restriction. This control measure significantly reduces or even off-highway. On the other hand, congress control ensures smooth merging of flows at conflict points. The control of exits affects the nature of the movement of vehicles flowing into the stream. Exit control methods are used to streamline the pace at which cars enter the highway.

There are 4 exit management strategies:

- restriction of access to the congress: temporary or permanent;

- control of exits using a traffic light object (Ramp metering) [1-4];

- Creation of traffic priorities for a special group of cars;

- Organization of queue management of vehicles on a ramp.

The strategy of traffic light control of congresses is well established at transport interchanges and at the entrances to cities. The presence of a traffic light object optimally supports the movement parameters at the exit for a smoother and more seamless flow merging. This is achieved by uniformly changing phases of regulation and the sequence of vehicles passing from the congress. Regulation of the exit also ensures that dense groups of vehicles that are approaching the freeway are broken down into individual cars, thereby avoiding confusion at the confluence points.

Exit control with the help of the Ramp metering traffic light facility - «motorway entry control system» - is an effective method of organizing traffic in many countries of the world. This system is used at two-level interchanges in conditions when a less intense flow of vehicles leaves one highway on a turning exit and tries to break into traffic on another highway.

In the Voronezh region, the level of motorization is increasing every year. During rush hours on major city highways leading from suburban areas to the city center, a drop in the performance of the road network occurs. As a rule, two-level interchanges with ramp exits are organized at the entrances to cities, along which the flow from one highway merges into traffic along another. In conditions of high intensity, such places are a potential «narrow neck», where the throughput decreases due to the random entry of the flow onto the highway. To optimize traffic on such sections of the highway, it is necessary to use intelligent transport systems that can solve problems with congestion at two-level junctions, and prevent traffic blocking as a result of a short circuit.

«The system of control of the entrance to the highway» («ramp metering») - regulation of exits [5]. This is an intelligent system designed to organize traffic, in which traffic is controlled at the exit at the point where it merges with the main traffic on the freeway in order to reduce the likelihood of traffic jams. For a smooth and unimpeded merger of flows, the traffic light object supports the parameters of movement at the exit, by rhythmically changing control phases and the alternate passage of vehicles from the exit. The pass is carried out so that the car is able to get into the interval formed between the simultaneously moving cars. Due to this, interruptions in traffic on the freeway are reduced, as drivers of the main stream have no reason to slow down and slow down when the vehicle is injected into the general traffic flow. In addition, the regulation of the congress ensures the breakdown of dense groups of vehicles traveling to the freeway into separate cars, which helps to avoid confusion at the point of confluence, the formation of a «narrow neck» and to exclude drivers from competing for gaps in the main stream. 
When implementing this system, a traffic light object is installed at the congress, a stop line is placed before entering the freeway and a warning sign is set in front of the congress, informing the driver about the presence of an adjustable section (fig. 1).

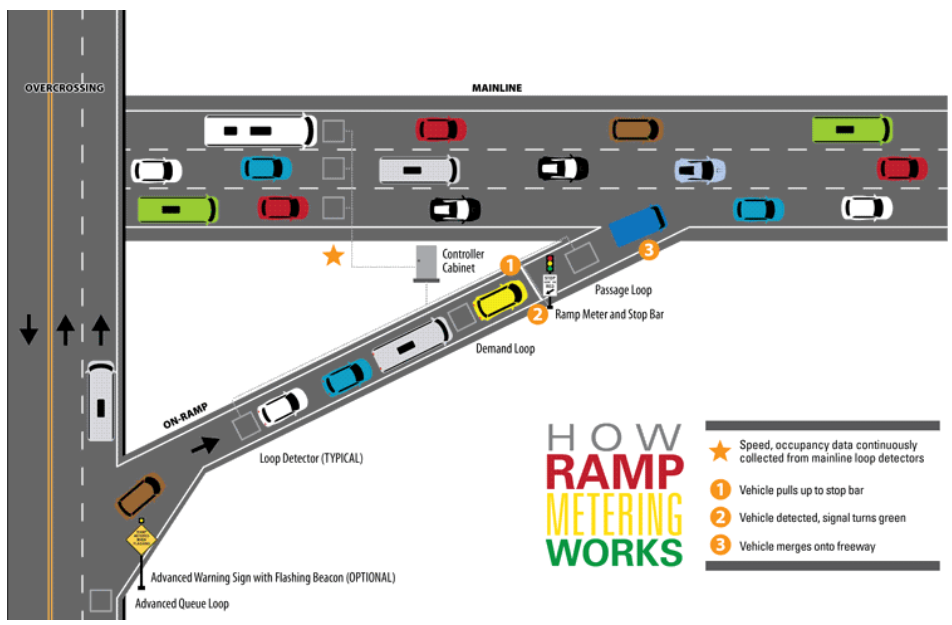

Fig. 1. An example of a congress regulation scheme

The standard scheme of congress regulation (fig. 1) also includes such elements as:

- Controller cabinet - control cabinet of a traffic light object;

- Passage loop - pass detector, which is installed behind the stop line, fixing the fact of the vehicle passing through the controlled area;

- Demand loop - a presence detector installed in front of the stop-line and traffic light, fixing the vehicle waiting for a green traffic signal;

- Loop detector - a presence detector, which is installed at the beginning of the congress, monitoring the queue of vehicles. In the event of a congestion, if the detector determines that the vehicle queue has reached the beginning of the exit, the «queue redefinition» algorithm starts, in which the controller increases the signal duration to the limit. Thus, the number of cars arriving is less than the number leaving the congress;

- Advanced Queue Loop - an additional queue detector, controls the length of the queue of cars;

- Advanced warning sign with flashing beacon - an additional road sign with a flashing beacon informing the driver about the presence of traffic lights at the congress [6].

\section{Smart entry implementation example}

To study and implement the described system, the team of authors selected a section of the road network - a tube-like interchange in Voronezh on January 9th street (Fig. 2), which directly leads to the city. The purpose of this study is to introduce a traffic light control system at this congress; for this event, at the first stages, it is necessary to study transport parameters: traffic flow intensity and time delays. Determine the peak values of the transport parameters in the direction of the regional center at the located points of entry along the main and secondary roads, and then proceed to the implementation of the intellectual exit. 


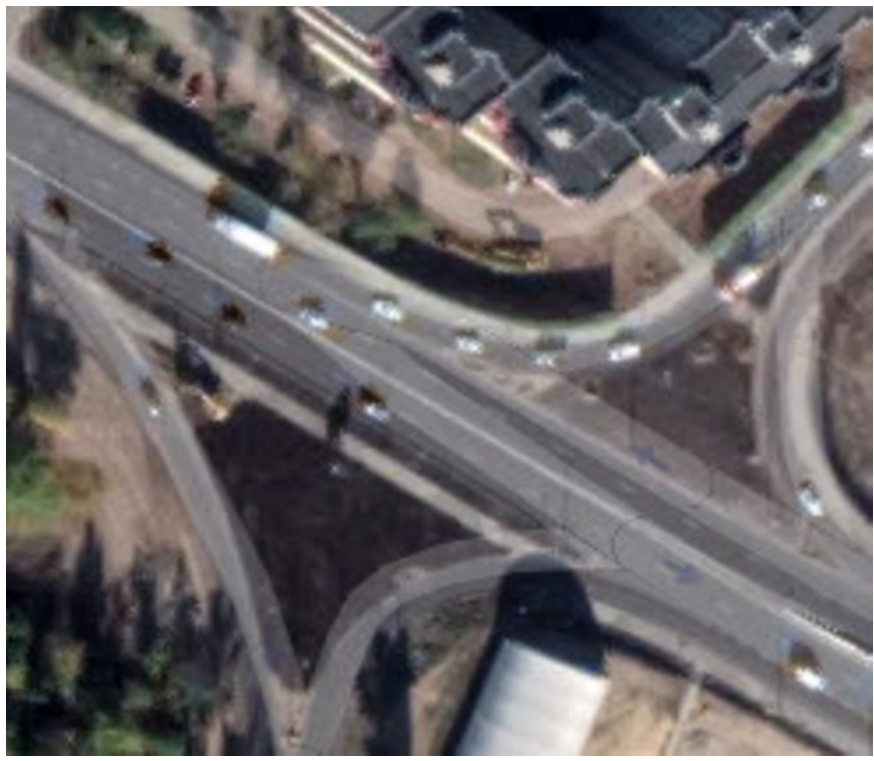

Fig. 2. The object of research of the city of Voronezh (satellite image)

The calculation of the number of cars was carried out at 6-minute intervals for an hour to get the nature of the intensity distribution over time. The reduced intensity on the highway at the exit from the crossing zone at rush hour was $N_{p}=114$ units/h, cars accounted for 95\% of the traffic. Reduced traffic on the ramp during rush hour $N_{p}=23$ units/h, $96 \%$ of the traffic flow are cars. Knowing the flow parameters at the exit from the intersection zone, we calculate the intensity and composition of the flow at the entrance to the exit. Reduced traffic intensity on the approach to the intersection at rush hour $N_{p}=91$ units/h, passenger cars account for $95 \%$ of the traffic flow. The analysis found that throughput is reduced in the merger zone by $15 \%$ due to flow on the ramp. The average transport delay per rush hour on the highway $t_{\Delta m}=39.7 \mathrm{~s}$, on the ramp $t_{\Delta p}=74.1 \mathrm{~s}$.

Before introducing the congress control system, it is necessary to determine the algorithm of the traffic light object. The local ALINEA algorithm is suitable for this section of the road network, since there are no other ramps in the section, the configuration of such a traffic interchange will not lead to the closure of flows, and this algorithm is adaptive [7]. We will configure the parameters for this algorithm: the location of the transport detector at the exit from the confluence zone from $40 \mathrm{~m}$ to $500 \mathrm{~m}$; correction parameter $\mathrm{K}_{\mathrm{R}}$; optimal load level $\mathrm{O}_{\text {opt}}$; cycle time (from $40 \mathrm{~s}$ to $5 \mathrm{~min}$ ).

Having determined the load levels during the studied period of time, we obtained the optimal load $\mathrm{O}_{\text {opt }}=0.31$. Knowing the indicators of the reduced intensity and the load level, we found the value of the regulation parameter - the rate at which vehicles are released onto the highway, according to formula (1) (table 1):

$$
r(k)=r(k-1)+K_{R} \cdot\left[O_{p t}-O_{k-1}\right]
$$

where $r(k)$ - is the control parameter at time k, unit/min; $r_{t}$ - is the control parameter at the previous time instant $k-1$, units/min; $K_{R}$ - correction parameter of regulation, unit/min; $O_{p t}$ the optimal level of load on the road; $O_{t+1}$ - load level at time $t+1$.

Reduced traffic on the ramp leads to an overall decrease in load on the highway. The algorithm keeps the load level at an optimal level. Total load per hour decreased by $10 \%$. 
Table 1. Regulation parameters

\begin{tabular}{|c|c|c|c|}
\hline Time interval k & $\begin{array}{c}\text { The reduced } \\
\text { intensity, units/6 min }\end{array}$ & $\begin{array}{c}\text { Existing load } \\
\text { level O }\end{array}$ & $\begin{array}{c}\text { Regulation parameter } \\
\mathrm{r}(\mathrm{k}) \text {, units/6 min }\end{array}$ \\
\hline 1 & 103 & 0,33 & 22 \\
\hline 2 & 115 & 0,36 & 22 \\
\hline 3 & 92 & 0,29 & 22 \\
\hline 4 & 142 & 0,45 & 19 \\
\hline 5 & 176 & 0,56 & 17 \\
\hline 6 & 202 & 0,64 & 13 \\
\hline 7 & 286 & 0,91 & 11 \\
\hline 8 & 158 & 0,50 & 11 \\
\hline 9 & 105 & 0,33 & 11 \\
\hline 10 & 114 & 0,36 & \\
\hline
\end{tabular}

The traffic intensity for the ramp is set by the parameter $r(k)$ based on the load level of the highway, which is implemented using traffic control. Since there is no traffic control at the congress, we will take the saturation flux $(S)$ equal to the throughput at the congress $P_{c}$, ( $S=P_{c}=769 \mathrm{unit} / \mathrm{h}$ ), and the duration of the green traffic light for a cycle duration of $60 \mathrm{~s}$ is calculated by formula 2 :

$$
g=\frac{r(k)}{S} \cdot T_{c}
$$

where $g$ - is the duration of the green traffic light, s; $S$ - is the saturation flow on the ramp, auto/h; $T_{c}$ - cycle time, s.

The green signal of the traffic light should turn on at that moment in time when a gap is formed in the right lane of the main stream. To detect gaps, it is proposed to use an algorithm based on the condition for providing a double dynamic dimension, according to formula $3[8,9]$ :

$$
L_{D}=l_{c}+v_{c} t_{r}+0,03 v_{c}^{2}+1
$$

where $l_{c}$ - is the length of the car, $\mathrm{m} ; v_{c}$ - vehicle speed, $\mathrm{m} / \mathrm{s} ; t_{r}$ - driver reaction time, sec.

Based on all the calculations, a flow gap search algorithm is compiled and the throughput part of the control cycle is set. This method is achieved by breaking the group of vehicles into single vehicles, thereby increasing the safety of merging flows [10-12].

\section{Conclusions}

Installation of this traffic light object is carried out in accordance with the standards of the Russian Federation. The controller cabinet should be placed in such a way as to exclude the possibility of a collision that goes beyond the carriageway of the vehicle with the cabinet, to ensure safe access for personnel to it and to ensure the visibility of traffic lights and roads. It is not recommended to place the cabinet in the area between the exit and the highway, as well as on the security island. Transport detectors should be placed on the main road at a distance of 40-500 meters from the "nose» of the congress. On each lane of the highway should be located 2 inductive transport detectors. The distance between them should be $6 \mathrm{~m}$ from edge to edge.

By introducing traffic light control under the control of the feedback algorithm, the existing load decreased by $10 \%$. The average time delay of one vehicle on a ramp increases by $27 \%\left(t_{\Delta p}=94.6 \mathrm{~s}\right)$. As a result of an increase in the waiting time for cars at the congress 
and ensuring their smooth merger, transport delays on the highways will decrease by $48 \%$ $\left(t_{\Delta m}=19.1 \mathrm{~s}\right)$.

\section{References}

1. T. Bellemans, B. De Moor, B. De Schutter, Model predictive control for ramp metering of motorway traffic: a case study. Control Engineering Practice 14 (2006)

2. P. Zheng, M. McDonald, Evaluation of effects of ramp metering on merging operations. Transportation Research Record 2012 (2007)

3. Q. Meng, H.L. Khoo, A pareto-optimization approach for a fair ramp metering Transportation Research Part C: Emerging Technologies 18 (2010)

4. A.M. Gorelov, A.A. Vlasov, Entrance management on highways Science and technology in the road industry $\mathbf{1}$ (2015)

5. B. Bellemans, De Schutter, G. Wets, B. De Moor. Model predictive control for ramp metering combined with extended kalman filter-based traffic state estimation. Proceedings of the IEEE Intelligent Transportation Systems Conference ITSC (2006)

6. Ramp Management and Control Handbook, Federal Highway Administration (2006)

7. M. Abdel-Aty, A. Dhindsa, V. Gayah, Considering various ALINEA ramp metering strategies for crash risk mitigation on freeways under congested regime. Transportation Research Part C: Emerging Technologies 15 (2007)

8. A.N. Novikov, I.A. Novikov, A.G. Shevtsova, A.G. Burlutskaya, Application of the Ramp Metering system at two-level interchanges The Russian Automobile and Highway Industry Journal 16 (2019)

9. A.G. Shevtsova, A.G. Burlutskaya, V.V. Vasilieva, Implementation of the Ramp Metering Intelligent Transport System on the Example of Belgorod World of Transport and Technological Machines 4 (2018)

10. A. Novikov, I. Novikov, A. Shevtsova, Study of the impact of type and condition of the road surface on parameters of signalized intersection Transportation Research Procedia (2018)

11. V.M. Vlasov, A.N. Novikov, I.A. Novikov, A.G. Shevtsova, Definition of perspective scheme of organization of traffic using methods of forecasting and modeling IOP Conference Series: Materials Science and Engineering Processing Equipment, Mechanical Engineering Processes and Metals Treatment (2018)

12. S.V. Dorokhin, V.A. Zelikov, Y.V. Strukov, D.V. Likhachev, A.N. Novikov, I.A. Novikov, A.G. Shevtsova, Investigation of methods for calculating duration of light signal regulation cycle Journal of Physics: Conference Series 1015 (2018) 